\title{
Disrupted intestinal structure in a rat model of intermittent hypoxia
}

\author{
JUNPING WU ${ }^{1,2^{*}}$, XIN SUN ${ }^{1,3^{*}}$, QI WU ${ }^{1,3}$, HONGWEI LI ${ }^{1,2}$, LI LI $^{2}$, JING FENG $^{4}$, SUBEI ZHANG ${ }^{1}$, \\ LONG XU ${ }^{1,3}, \mathrm{KUAN} \mathrm{LI}^{1,3}, \mathrm{XUE} \mathrm{LI}^{1,3}, \mathrm{XING} \mathrm{WANG}^{2}$ and HUAIYONG CHEN ${ }^{1,3}$ \\ ${ }^{1}$ Department of Basic Medicine, Haihe Clinical College, Tianjin Medical University, Tianjin 300350; \\ ${ }^{2}$ Department of Respiratory Medicine, Tianjin Haihe Hospital; ${ }^{3}$ Key Research Laboratory for Infectious Disease Prevention \\ for State Administration of Traditional Chinese Medicine, Tianjin Institute of Respiratory Diseases, Tianjin Haihe Hospital, \\ Tianjin 300350; ${ }^{4}$ Respiratory Department, Tianjin Medical University General Hospital, Tianjin 300052, P.R. China
}

Received May 25, 2015; Accepted March 9, 2016

DOI: $10.3892 / \mathrm{mmr} .2016 .5068$

\begin{abstract}
Obstructive sleep apnea (OSA) is a chronic condition characterized by chronic intermittent hypoxia (IH) and subsequent reoxygenation (ROX). The gastrointestinal system, which is particularly sensitive to tissue hypoxia and reduced perfusion, is likely to be affected by OSA. A rat model of IH was used to analyze oxidative stress-associated genes and tight junction proteins by reverse transcription-quantitative polymerase chain reaction. Subsequently, altered morphology of the duodenal mucosa and elevated Chiu scores were observed in the IH-exposed rats. In addition, IH exposure resulted in upregulation of the nicotinamide adenine dinucleotide phosphate (NADPH) oxidase subunits, NADPH oxidase 2 and p22phox, in the small intestine, and upregulation of transcription factors, including hypoxia-inducible factor-1, nuclear factor $-\kappa \mathrm{B}$ and activator protein-1. Furthermore, the mRNA expression levels of intestinal tight junction (TJ)-related proteins, claudin-1 and claudin-4, were decreased in the IH-exposed group, as compared with in the control group. In conclusion, the present study demonstrated that OSA, which is characterized by IH and ROX, may lead to disruption of the duodenum. The mechanism underlying the
\end{abstract}

Correspondence to: Professor Qi Wu, Key Research Laboratory for Infectious Disease Prevention for State Administration of Traditional Chinese Medicine, Tianjin Institute of Respiratory Diseases, Tianjin Haihe Hospital, 890 Jingu Road, Tianjin 300350, P.R. China

E-mail:wq572004@163.com

Dr Huaiyong Chen, Department of Basic Medicine, Haihe Clinical College, Tianjin Medical University, 890 Jingu Road, Tianjin 300350, P.R. China

E-mail: huaiyong.chen@foxmail.com

*Contributed equally

Key words: intermittent hypoxia, duodenum, hypoxia-inducible factor-1 $\alpha$, tight junction, nuclear factor- $\mathrm{kB}$ effects of OSA on duodenal morphology may be associated with increased oxidative stress and activation of transcription factors, subsequently inducing intestinal TJ disruption and intestinal injury.

\section{Introduction}

Obstructive sleep apnea (OSA) is a breathing disorder that is characterized by repetitive episodes of complete or partial upper airway obstruction during sleep, which leads to intermittent reduction or complete blockage of airflow (1). The prevalence of OSA is $3-7 \%$ in men and $2-5 \%$ in women (1). Repetitive OSA results in chronic intermittent hypoxia (IH), which is followed by reoxygenation (ROX), and is characterized by frequent decreases in blood $\mathrm{O}_{2}$ saturation. The clinical symptoms of sleep apnea were reported as early as the $19^{\text {th }}$ century (2); however, it was not until the 1980 s that researchers began to investigate and understand OSA (3).

OSA has been associated with numerous comorbidities, including cardiovascular alterations, diabetes and depression (4). Although efforts have been made to comprehend the consequences of OSA and the underlying state of IH, there may be more problems or comorbidities associated with OSA than originally expected. The gastrointestinal system is likely to be affected by OSA, since the gastrointestinal epithelium is particularly sensitive to tissue hypoxia and reduced perfusion. Furthermore, a clinical study involving 35,480 patients indicated sleep apnea as an independent risk factor for gastric and duodenal ulcer bleeding (5), thus suggesting that OSA may compromise the gastrointestinal system; however, the underlying mechanism is not well understood. Therefore, the present study hypothesized that $\mathrm{IH}$, a characteristic of OSA, may induce intestinal injury.

Integrity of the intestinal epithelium is essential for normal physiological function and the prevention of disease, since it restricts the free passage of toxic and infectious molecules from the gut lumen whilst allowing selective paracellular absorption of nutritive material. The major determinants of intestinal barrier function are the intercellular tight junctions (TJs), which are located in the uppermost region of the lateral membranes of epithelial and endothelial cells (6). Several TJ 
compounds have been identified, including the transmembrane proteins occludin and claudins, and the peripheral membrane proteins zonula occludens (ZOs) (7). Claudins are considered integral proteins of TJs that regulate size selectivity of the TJ barrier. Occludin is thought to be the primary sealing protein of the epithelial intercellular space, whereas ZOs are the critical scaffold proteins that link transmembrane TJ components to the intracellular actin cytoskeleton (8).

Growing evidence from cellular and animal models, and population surveys of OSA, has demonstrated that exposure to $\mathrm{IH}$ is associated with the activation of oxidative stress and inflammatory processes (9). IH induces the accumulation of reactive oxygen species (ROS), which initiates oxidative stress-sensitive signaling pathways and inflammatory processes. Various transcription factors and inflammatory mediators implicated in this process have previously been identified (10). Among these, much attention has been focused on hypoxia-inducible factor-1 (HIF-1), nuclear factor- $\kappa \mathrm{B}$ $(\mathrm{NF}-\mathrm{\kappa B})$ and activator protein-1 (AP-1). The transcription factor HIF-1 is the main regulator of oxygen homeostasis and serves a key role in the response to hypoxia in most tissues (11). NF- $\mathrm{KB}$ and AP-1 are transcription factors implicated in inflammatory processes. Once they are activated, several target genes are transcribed, triggering an inflammatory cascade. A previous study suggested that the expression and function of TJs are affected by proinflammatory cytokines and intracellular signaling molecules (12). Therefore, it is essential to identify whether IH has an effect on the intestinal gene expression of transcription factors and inflammatory mediators, and whether it induces TJ disruption via activation of oxidative stress and inflammatory processes.

In the present study, a rat model was developed to mimic the recurrent IH and subsequent ROX experienced by patients with OSA. It was hypothesized that this pathological environment may result in activation of oxidative stress and inflammatory processes in the duodenum, subsequently compromising intestinal barrier function by disrupting TJs.

\section{Materials and methods}

Ethics statement. Rats were used in strict accordance with the protocol approved by the Animal Care Committee of Tianjin Medical University General Hospital (Tianjin, China).

Animals and treatments. Male Wistar rats $(180 \pm 20 \mathrm{~g} ; \mathrm{n}=30$; 6-weeks-old) were purchased from the Model Animal Center of Radiological Medicine Research Institute, China Academy of Medical Science (Tianjin, China). Rats were housed in standard laboratory cages $(n=5 /$ cage $)$ at $22^{\circ} \mathrm{C}$ with a $12 \mathrm{~h}$ light/dark cycle and free access to food and water. The rats were randomly divided into two groups ( $n=15 /$ group) matched for body weight: The IH-exposed group and the control group. Rats in the IH-exposed group were exposed to $\mathrm{IH}$ for $8 \mathrm{~h}$ /day during the rodent diurnal sleep period, between 9 AM and $5 \mathrm{PM}$, repeatedly for 7 days/week for 8 consecutive weeks, in a specialized plexiglas chamber (dimensions 30x20x20 cm), as previously described (13). Pure nitrogen and compressed air were flushed into the chamber in turn to maintain an $\mathrm{IH}$ cycle. Each cycle of IH lasted $120 \mathrm{sec}$, the first $30 \mathrm{sec}$ being the hypoxic phase and the following $90 \mathrm{sec}$ the ROX phase (during which the nitrogen was replaced with clean air). Gas flow was regulated by timer-controlled solenoid valves and an $\mathrm{O}_{2}$ flow meter. The $\mathrm{O}_{2}$ and $\mathrm{CO}_{2}$ concentrations were continuously monitored by an $\mathrm{O}_{2}$ and $\mathrm{CO}_{2}$ concentration monitor (Hamilton Medical AG, Bonaduz, Switzerland). The control rats underwent an identical protocol; however, the nitrogen source was replaced with a clean air source.

Histological analysis. Following treatment, rats were anesthetized by intraperitoneal injection of $30 \mathrm{mg} / \mathrm{kg}$ pentobarbital sodium (Sigma-Aldrich, St. Louis, MO, USA), and the duodenum was excised and rinsed in ice-cold phosphate-buffered saline ( $\mathrm{pH}$ 7.4). The duodenal tissues were subsequently fixed in $10 \%$ neutral buffered formalin for $24 \mathrm{~h}$, were paraffin-embedded, cut into $5 \mu \mathrm{m}$ sections, and were processed for hematoxylin and eosin (H\&E) staining (Solarbio Science \& Technology Co., Ltd., Beijing, China). The stained sections were analyzed, and images of the representative fields were captured using an Olympus BX53 microscope (Olympus Corporation, Tokyo, Japan). Morphological injury of the duodenal mucosa was assessed using the Chiu histological injury scoring system for intestinal villi. The numerical scores were as follows: 0, normal mucosa; 1, development of subepithelial Gruenhagen's space and vacuolization at the apex of the villi; 2, extension of the subepithelial space with moderate lifting of the epithelial layer from the lamina propria; 3, massive subepithelial lifting down the sides of villi; 4 , epithelial lifting and vacuolization from the tip to the lower portion of villi; and 5, mucosal hemorrhage, ulceration and disintegration of the lamina propria (14). Two independent and blinded researchers performed the histological scoring.

Total RNA isolation. TRIzol ${ }^{\circledR}$ reagent (Invitrogen; Thermo Fisher Scientific, Inc., Waltham, MA, USA) was used to extract RNA from homogenized duodenal tissues, according to the manufacturer's protocol. Extract yield and quality were determined by measuring the absorbance at 260 and $280 \mathrm{~nm}$ using a MaestroNano Micro-volume Spectrophotometer (Maestrogen, Inc., Las Vegas, NV, USA). The absorbance ratio of 260:280 nm was between 1.8 and 2.0.

Reverse transcription-quantitative polymerase chain reaction $(R T-q P C R)$. mRNA $(3 \mu \mathrm{g})$ was reverse transcribed into cDNA with an oligo (dT) primer for $1 \mathrm{~h}$ at $50^{\circ} \mathrm{C}$ using the TIANScript RT kit (Tiangen Biotech Co., Ltd., Beijing, China), according to the manufacturer's protocol. RT-qPCR was performed using iQ SYBR Green Supermix (\#1708880; Bio-Rad Laboratories, Inc.,Hercules, CA, USA) with a reaction volume of $20 \mu \mathrm{l}$, according to the manufacturer's protocol. Gene-specific primers were designed using the Primer-Quest SM software (sg.idtdna.com/Primerquest/Home/Index; Integrated DNA Technologies, Inc., Coralville, IA, USA), and were commercially produced by BGI Tech (BGI Tech Solutions Co., Ltd., Shenzhen, China). Primer sequences are listed in Table I. DNA amplification was carried out using a CFX96 Touch Real-Time PCR Detection system (Bio-Rad Laboratories, Inc.) with the following reaction conditions: Initial heating cycle at $95^{\circ} \mathrm{C}$ for $2 \mathrm{~min}$; followed by 40 cycles alternating between denaturation at $95^{\circ} \mathrm{C}$ for $25 \mathrm{sec}$, primer annealing at $60^{\circ} \mathrm{C}$ for $25 \mathrm{sec}$, and extension at $72^{\circ} \mathrm{C}$ for $20 \mathrm{sec}$. 
Table I. DNA primer sequences for reverse transcription-quantitative polymerase chain reaction.

\begin{tabular}{lll}
\hline Gene & \multicolumn{1}{c}{ Forward primer } & Reverse primer \\
\hline GAPDH & 5'-TGGAGTCTACTGGCGTCTTC-3' & 5'-TTCACACCCATCACAAACATG-3' \\
Nox2 & 5'-GGCTGTGAATGAGGGACTC-3' & 5'-CCAGTGCTGACCCAAGAAG-3' \\
p22phox & 5'-AAGTACCTGACCGCTGTGG-3' & 5'-AGGTAGATCACACTGGCAATG-3' \\
HIF-1 $\alpha$ & 5'-AAGAAACCGCCTATGACGTG-3' & 5'-CCACCTCTTTTTGCAAGCAT-3' \\
NF-kB & 5'-AGCCCTATGCCTTTTCAACAT-3' & 5'-GTCCAGGGAGGTCACAGA-3' \\
c-fos & 5'-CGAAGGGAAAGGAATAAGA-3' \\
Claudin-1 & 5'-TGTCCACCATTGGCATGAAG-3' & 5'-GCCACTAATGTCGCCAGACC-3' \\
Claudin-2 & 5'-ACAGCACTGGCATCACCCA-3' & 5'-GCGAGGACATTGCACTGGAT-3' \\
Claudin-4 & 5'-AAGGCCAAGGTCATGATCACAG-3' & 5'-GAAGTCGCGGATGACGTTGT-3' \\
Occludin & 5'-CTACTCCTCCAACGGCAAAG-3' & 5'-AGTCATCCACGGACAAGGTC-3' \\
ZO-1 & 5'-ATTCAGTTCGCTCCCATGAC-3' & 5'-GCTGTGGAGACTGTGTGGAA-3'
\end{tabular}

GAPDH, glyceraldehyde 3-phosphate dehydrogenase; Nox2, nicotinamide adenine dinucleotide phosphate-oxidase; HIF-1 $\alpha$, hypoxia-inducible factor- $1 \alpha$; NF- $\kappa \mathrm{B}$, nuclear factor- $\kappa \mathrm{B}$; ZO-1, zona occludens-1.

A final extension step at $72^{\circ} \mathrm{C}$ for 10 min was conducted. The housekeeping gene, glyceraldehyde 3-phosphate dehydrogenase (GAPDH), was used as an internal control. Melting curves were used to identity the amplicons. Relative mRNA expression levels of the target genes were calculated using the $2^{-\Delta \Delta \mathrm{Cq}}$ method, and were normalized to the levels of GAPDH in the same sample (15).

Statistical analysis. Results are presented as the mean \pm standard error of the mean and experiments were repeated three times. The data were analyzed using SPSS software, version 13.0 (SPSS, Inc., Chicago, IL, USA) and differences between paired groups were analyzed using Student's t-test. $\mathrm{P}<0.05$ was considered to indicate a statistically significant difference.

\section{Results}

Exposure to IH results in damage to the duodenal epithelium. Hypoxia is known to lead to inflammation; in order to assess whether IH contributes toward injury to the duodenal epithelium, duodenal morphology was examined. Evaluation of the H\&E-stained sections revealed morphological alterations to the duodenal mucosa in response to $\mathrm{IH}$ exposure (Fig. 1A and B). High-power images of the general epithelial structures of the duodenum from the control or IH-exposed rats were captured (Fig. 1C and D). The histological images of the duodenal specimens from the control rats (Fig. 1A) exhibited normal-appearing mucosal villi with consistent mucosa, as compared with the IH-exposed rats (Fig. 1B). IH-exposed rats exhibited disintegration of the mucosal villi and infiltration of inflammatory cells (Fig. 1B). Furthermore, necrosis and superficial ulceration were detected in the mucosa of certain IH-exposed rats (data not shown). The villous injury score of the IH-exposed rats (mean injury score, $4.00 \pm 0.63)$ was markedly higher compared with the control rats (mean injury score, $0.67 \pm 0.58$; Fig. 1E). These findings suggest that exposure to IH may result in marked pathophysiological alterations in duodenal tissue.
IH exposure induces activation of oxidative stress and transcription factor expression. A previous study indicated that recurrent hypoxia and ROX cycles increase the production of ROS in OSA (16). Nicotinamide adenine dinucleotide phosphate (NADPH) oxidase serves a key role in oxidative stress and is an enzyme involved in the production of ROS (9). To examine whether IH affects NADPH oxidase activity in the intestine, and if so, whether NADPH oxidase activation contributes to the expression of IH-induced transcription factors, the expression levels of NADPH oxidase subunit genes were measured in the IH-exposed and control rats. There was a significant increase in the mRNA expression levels of the NADPH oxidase subunits NADPH oxidase 2 (Nox2) (Fig. 2A; P=0.003) and p22phox (Fig. 2B; $\mathrm{P}=0.005$ ) in the IH-exposed rats. These data suggest an overexpression of NADPH oxidase in the IH-exposed rats. Therefore, it may be hypothesized that NADPH oxidase is a major source of ROS in the IH-exposed duodenum, and that upregulation of NADPH oxidase results in increased ROS, thereby mediating the onset of oxidative stress.

HIF-1 is a heterodimeric protein that is composed of an $\mathrm{O}_{2}$-regulated HIF-1 $\alpha$ subunit and a constitutively expressed HIF-1 $\beta$ subunit. Hypoxia induces upregulation of HIF-1, and the activity of HIF-1 is primarily determined by the HIF-1 $\alpha$ subunit. To examine whether IH activated HIF-1, the mRNA expression levels of HIF-1 $\alpha$ were assessed. Compared with the control group, a significant increase in the mRNA expression levels of HIF-1 $\alpha$ was detected in the IH group (Fig. 2C; $\mathrm{P}=0.014$ ).

AP-1 is a protein complex formed by the protein products of immediate early genes, including c-fos and c-jun. Activation of AP-1 is usually indirect and represented by c-fos mRNA expression levels. The mRNA expression levels of c-fos (Fig. 2D; $\mathrm{P}=0.033$ ) were significantly increased in the IH-exposed rats. In addition, an increase in the mRNA expression levels of $\mathrm{NF}-\kappa \mathrm{B}$ was detected in the duodenum of the $\mathrm{IH}$-exposed rats (Fig. 2E; $\mathrm{P}=0.07$ ). These data indicate that IH may activate transcription factors in the duodenum. 

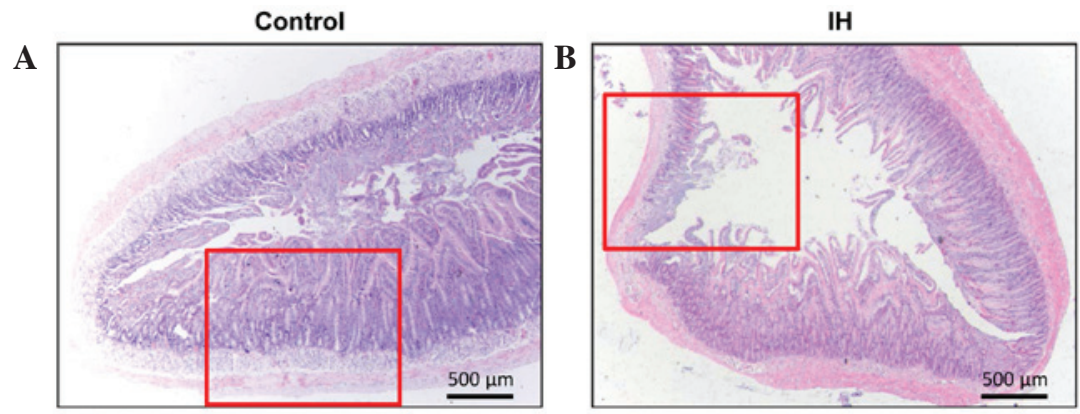

C

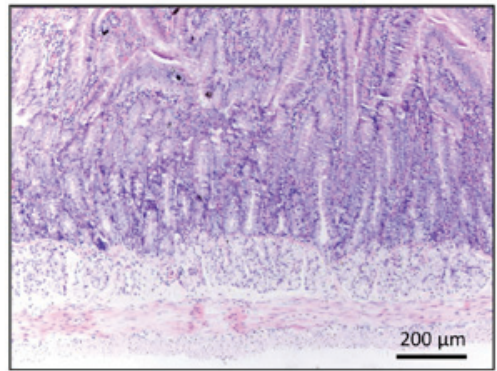

D

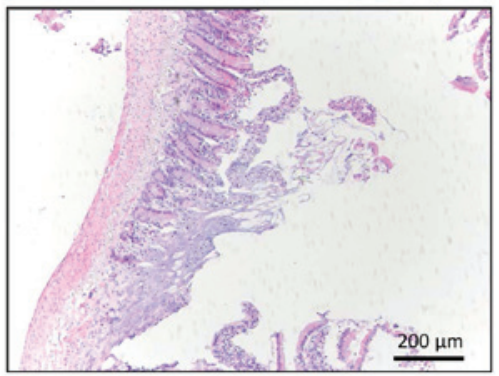

E

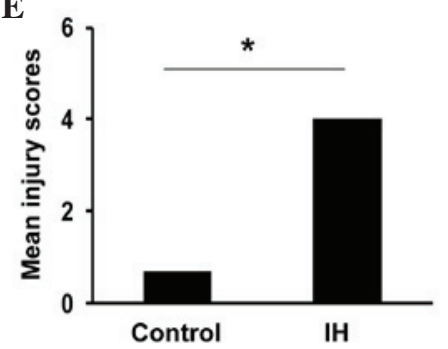

Figure 1. Disrupted duodenal epithelium in IH-exposed rats. The duodenum was collected from (A) control and (B) IH-exposed (8 weeks exposure) rats. An equivalent area of duodenal tissue was excised from the control or IH-exposed rats, and the tissues were fixed and stained with hematoxylin and eosin. (C and D) High-power view of general duodenal epithelial structures from the control and IH-exposed rats, respectively. (E) Mean villous injury scores of the control and $\mathrm{IH}$-exposed rats. Data are presented as the mean \pm standard error of the mean. ${ }^{*} \mathrm{P}<0.05$ vs. control. IH, intermittent hypoxia.

IH exposure selectively regulates the $m R N A$ expression levels of TJ proteins. Due to the key function of TJ proteins in the integrity of intestinal mucosa, the present study examined whether IH exposure regulated TJ components in the duodenum, including claudin-1, -2, -4, occludin and ZO-1. RT-qPCR demonstrated that the mRNA expression levels of claudin-1 (Fig. 3A) and claudin-4 (Fig. 3B) were significantly reduced by $\mathrm{IH}$ exposure compared with the control group $(\mathrm{P}<0.01$ and $\mathrm{P}<0.05$, respectively). However, no significant alterations were detected in claudin-2, occludin or ZO-1 mRNA expression (Fig. 3C-E; P>0.05). These data suggest that IH exposure selectively loosens TJ proteins of the intestinal luminal cells to increase intestinal permeability, which subsequently leads to a breach in the mucosal barrier during IH.

\section{Discussion}

The present study used a rat model to provide evidence that $\mathrm{IH}$ exposure, the hallmark feature of OSA, may lead to disruption in the duodenum. In addition, increased mRNA expression levels of oxidative stress-related genes and transcription factors were detected in the duodenum following exposure to IH.

IH and subsequent ROX are characteristics of OSA, which is similar to ischemia/reperfusion (I/R) injury. Although no direct study has observed intestinal injury in OSA, it has previously been reported that intestinal damage occurs following $I / R$ injury (17). Intestinal morphological injury alongside a raised Chiu score has been observed in response to I/R injury (17). In addition, functional studies of intestinal barrier function have demonstrated that intestinal permeability increases following I/R injury $(18,19)$. Conversely, no previous studies have reported a direct link between IH and intestinal injury. The present study demonstrated that the intestinal mucosa was significantly compromised following IH exposure, as evidenced by morphological alterations to intestinal structures and elevated Chiu scores. It may therefore be hypothesized that these changes increase mucosal permeability, leading to intestinal barrier dysfunction.

IH-induced oxidative stress represents a pathological link between OSA and resultant multiple organ injury. A previous study demonstrated that $\mathrm{IH}$ induces severe oxidative stress in the myocardium, brain, carotid body, adrenal gland and liver in animal models (20). Excess ROS may lead to radical-induced oxidation and damage, serving as key activator for transcription factors and inflammatory pathways (11). Cell culture and animal model studies have demonstrated that HIF-1 is activated by $\mathrm{IH}$ exposure $(21,22)$, due to the increased generation of ROS via activated NADPH oxidase and the resultant changes in intracellular $\mathrm{Ca}^{2+}$ (23). The present study demonstrated that HIF-1 $\alpha$ mRNA expression was upregulated in the duodenum following IH exposure. A previous study demonstrated the feed-forward interactions between HIF-1 and ROS under IH conditions (24). IH may activate HIF-1 via a ROS-dependent manner, whereas antioxidants prevent HIF-1 activation (25). Conversely, HIF-1 is required for IH-induced ROS generation, that is, IH elevates ROS levels in wild-type mice, but not in HIF-1 $\alpha$-deficient mice (26). These results suggested that IH may initially induce an increase in ROS levels by activating NADPH oxidase, which upregulates HIF-1 $\alpha$, and once HIF-1 is activated, it may further promote increases in ROS.

$\mathrm{NF}-\kappa \mathrm{B}$ and AP-1 are transcription factors, which have been investigated in $\mathrm{IH}$. The classical $\mathrm{NF}-\kappa \mathrm{B}$ pathway is thought to be activated by ROS. Previous studies have reported that $\mathrm{IH}$ induces activation of $\mathrm{NF}-\kappa \mathrm{B}$ and upregulation of $\mathrm{NF}-\kappa \mathrm{B}$-dependent genes (26), which is mediated via activation of p38 mitogen-activated protein (MAP) kinase (27). In addition, increased protein and mRNA expression levels of c-fos have been detected in animal and cell models following 
A

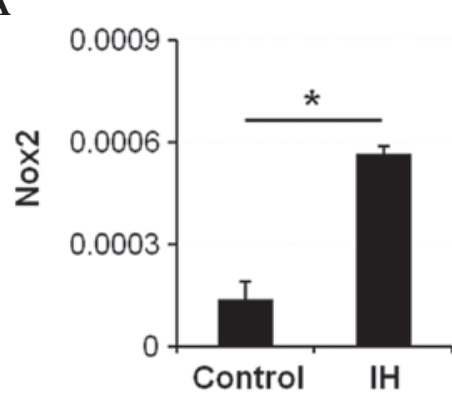

B

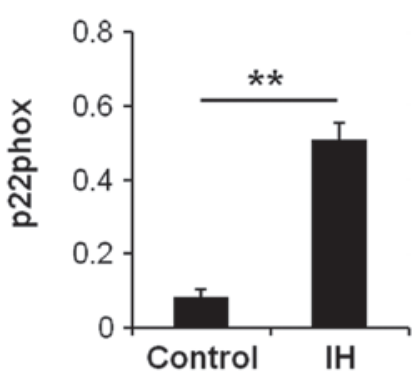

C

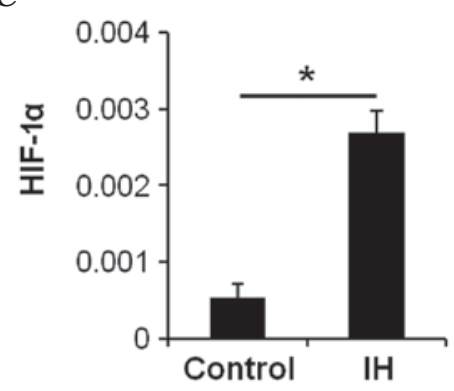

D

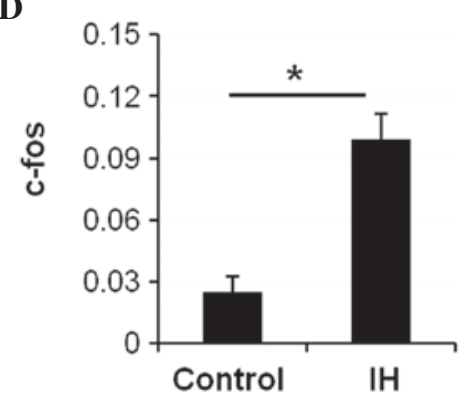

$\mathbf{E}$

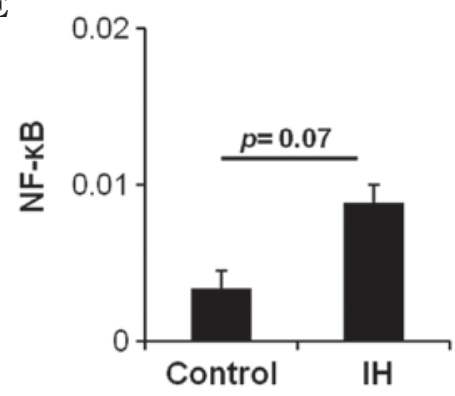

Figure 2. Expression of oxidative stress-related genes and transcription factors in the rat duodenum following IH exposure. mRNA expression levels of

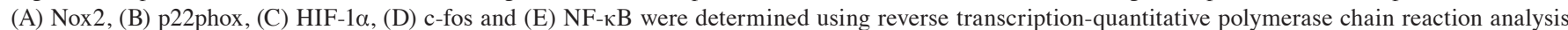
of duodenal tissue from control and IH-exposed rats. The mRNA expression levels were normalized to glyceraldehyde 3-phosphate dehydrogenase levels. Data are presented as the mean \pm standard error of the mean. ${ }^{*} \mathrm{P}<0.05$ and ${ }^{* *} \mathrm{P}<0.01$ vs. the control rats. IH, intermittent hypoxia; Nox 2 , nicotinamide adenine dinucleotide phosphate oxidase 2 ; HIF-1 $\alpha$, hypoxia-inducible factor- $1 \alpha$; NF- $\kappa \mathrm{B}$, nuclear factor $\kappa \mathrm{B}$.

A

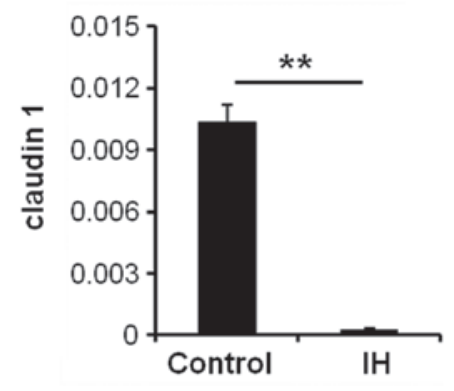

D

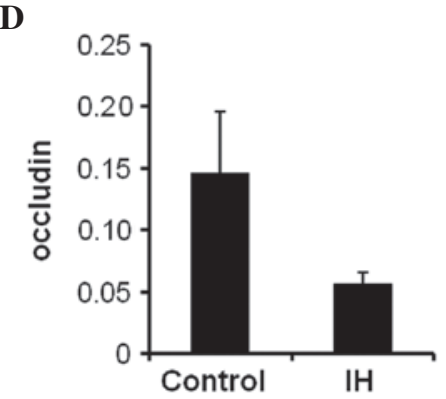

B

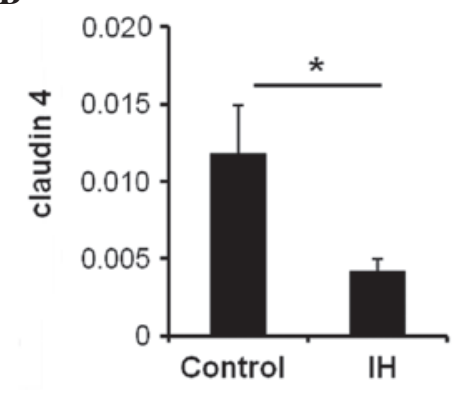

C

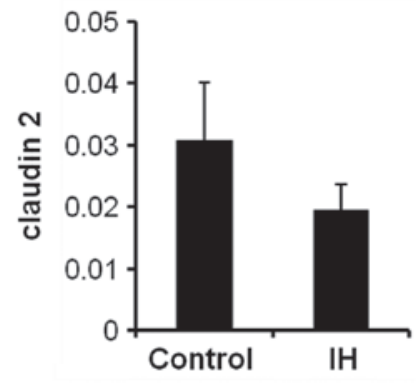

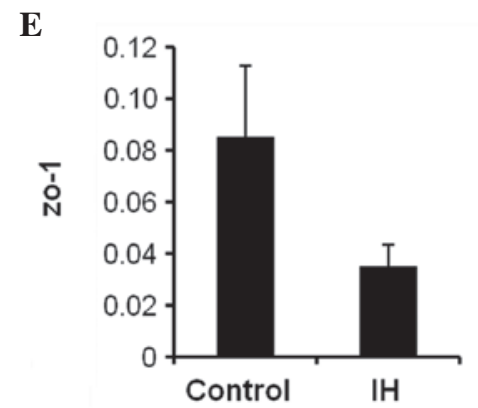

Figure 3. mRNA expression levels of tight junction proteins in the rat duodenum following IH exposure. The duodenal mRNA expression levels of (A) claudin-1, (B) claudin-4, (C) claudin-2, (D) occludin and (E) ZO-1 in the control and IH-exposed rats were determined using reverse transcription-quantitative polymerase chain reaction analysis, and were normalized to glyceraldehyde 3-phosphate dehydrogenase levels. Data are presented as the mean \pm standard error of the mean. ${ }^{*} \mathrm{P}<0.05$ and ${ }^{* *} \mathrm{P}<0.01$ vs, the control rats. IH, intermittent hypoxia; ZO-1, zona occludens- 1 .

exposure to IH (28), thus suggesting that AP-1 serves an important role in $\mathrm{IH}$.

TJs are important for maintaining integrity of the intestinal barrier (29). Disruption of TJs and increased paracellular permeability serve a role in the pathogenesis of several intestinal diseases (30). Furthermore, TJ proteins may be influenced by numerous transcription factors, including HIF-1. A previous study on HIF-1 $\beta$ knockdown cells detected significantly reduced levels of claudin-1, which subsequently led to increased intestinal permeability (31). However, the roles of HIF-1 $\alpha$ in the regulation of barrier integrity seem controversial. In addition, HIF-1 has been identified as a 
factor associated with barrier protection under hypoxic conditions (32). The present study demonstrated that HIF-1 may serve a gut-injurious role in IH-induced intestinal injury, since the expression of TJ-related proteins was upregulated.

The $N F-\kappa B$ signaling pathway has a role in intestinal epithelial homeostasis and repair (29), and disruption or anomalous activation of $\mathrm{NF}-\kappa \mathrm{B}$ may exaggerate the inflammatory response (33). A previous cell culture study demonstrated that TNF- $\alpha$ induced downregulation of claudin-1, -2, -4, and occludin, which could be partially alleviated via pharmacological inhibition of $N F-\kappa B$ (34). Furthermore, the NF- $\kappa \mathrm{B}$ signaling pathway has been reported to mediate increased expression of myosin light chain kinase, which induces opening of intestinal TJ proteins, thus resulting in TJ barrier breakdown (35). Activation of NF- $\kappa \mathrm{B}$ may also mediate claudin-1 internalization and increase paracellular permeability (36). Furthermore, NF- $\kappa$ B associates with AP-1 to induce redistribution of intestinal TJ permeability via increased MAP kinase phosphorylation (37) and interleukin-6 secretion (38). Taken together, these data demonstrate that $\mathrm{NF}-\kappa \mathrm{B}$ and AP-1 may disrupt intestinal epithelium by regulating TJ components.

Increasing evidence has illustrated the association between hypoxia and gastrointestinal disease $(39,40)$. The absorptive and barrier functions of the intestinal epithelium may be physiologically regulated by the availability of oxygen (39). It is well known that hypoxia may induce inflammation, and conversely, inflamed lesions often become severely hypoxic (41). In addition, hypoxia influences innate and adaptive immunity via activation of HIF-1 $\alpha$ (42). Therefore, it may be suggested that hypoxia is a significant component of the inflammatory microenvironment within the intestinal mucosa (40).

The present study has certain limitations. Constrained to the experimental technique, the present study failed to detect ROS accumulation directly. In addition, future experiments that analyze the expression levels of proteins associated with intestinal TJs and transcription factors by western blotting or immunohistochemistry are required.

In conclusion, the major observation of the present study is that OSA, characterized by $\mathrm{IH}$ and subsequent ROX, may cause disruption of the duodenum. The mechanism underlying the effects of OSA on duodenal morphology is associated with increased oxidative stress and activation of transcription factors, which may subsequently induce intestinal TJ disruption and intestinal injury. These data may provide a novel insight into the clinical treatment of patients with OSA, but intestinal complications should be kept in mind and caution taken to avoid these.

\section{Acknowledgements}

The present study was supported by the National Natural Science Foundation of China (grant nos. 31471121 and 81270144) and the Natural Science Foundation of Tianjin City (grant nos. 13JCYBJC22400, 13JCYBJC40000 and 14JCYBJC25700).

\section{References}

1. Lurie A: Obstructive sleep apnea in adults: Epidemiology, clinical presentation, and treatment options. Adv Cardiol 46: 1-42, 2011.
2. Dempsey JA, Veasey SC, Morgan BJ and O'Donnell CP: Pathophysiology of sleep apnea. Physiol Rev 90: 47-112, 2010.

3. Punjabi NM: The epidemiology of adult obstructive sleep apnea. Proc Am Thorac Soc 5: 136-143, 2008.

4. Kendzerska T, Mollayeva T, Gershon AS, Leung RS, Hawker G and Tomlinson G: Untreated obstructive sleep apnea and the risk for serious long-term adverse outcomes: A systematic review. Sleep Med Rev 18: 49-59, 2014.

5. Shiao TH, Liu CJ, Luo JC, Su KC, Chen YM, Chen TJ, Chou KT, Shiao GM and Lee YC: Sleep apnea and risk of peptic ulcer bleeding: A nationwide population-based study. Am J Med 126: 249-255.e1, 2013.

6. Tsukita S, Furuse $M$ and Itoh M: Multifunctional strands in tight junctions. Nat Rev Mol Cell Biol 2: 285-293, 2001.

7. Zahraoui A, Louvard D and Galli T: Tight junction, a platform for trafficking and signaling protein complexes. J Cell Biol 151: F31-F36, 2000.

8. Mitic LL, Van Itallie CM and Anderson JM: Molecular physiology and pathophysiology of tight junctions I. Tight junction structure and function: Lessons from mutant animals and proteins. Am J Physiol Gastrointest Liver Physiol 279: G250-G254, 2000.

9. Lurie A: Inflammation, oxidative stress, and procoagulant and thrombotic activity in adults with obstructive sleep apnea. Adv Cardiol 46: 43-66, 2011.

10. Prabhakar NR: Oxygen sensing during intermittent hypoxia: cellular and molecular mechanisms. J Appl Physiol (1985) 90: 1986-1994, 2001.

11. Bonsignore MR and Eckel J: ERS Meeting Report. Metabolic aspects of obstructive sleep apnoea syndrome. Eur Respir Rev 18: 113-124, 2009.

12. González-Mariscal L, Tapia R and Chamorro D: Crosstalk of tight junction components with signaling pathways. Biochim Biophys Acta 1778: 729-756, 2008.

13. Feng J, Wang QS, Chiang A and Chen BY: The effects of sleep hypoxia on coagulant factors and hepatic inflammation in emphysematous rats. PLoS One 5: e13201, 2010.

14. Chiu CJ, McArdle AH, Brown R, Scott HJ and Gurd FN: Intestinal mucosal lesion in low-flow states. I. A morphological, hemodynamic, and metabolic reappraisal. Arch Surg 101: 478-483, 1970.

15. Livak KJ and Schmittgen TD: Analysis of relative gene expression data using real-time quantitative PCR and the 2(-Delta Delta C(T)) Method. Methods 25: 402-408, 2001.

16. Lavie L: Obstructive sleep apnoea syndrome - an oxidative stress disorder. Sleep Med Rev 7: 35-51, 2003.

17. Zheng X, Mao Y, Cai J, Li Y, Liu W, Sun P, Zhang JH, Sun X and Yuan $\mathrm{H}$ : Hydrogen-rich saline protects against intestinal ischemia/reperfusion injury in rats. Free Radic Res 43: 478-484, 2009.

18. Yang R, Gallo DJ, Baust JJ, Watkins SK, Delude RL and Fink MP: Effect of hemorrhagic shock on gut barrier function and expression of stress-related genes in normal and gnotobiotic mice. Am J Physiol Regul Integr Comp Physiol 283: R1263-R1274, 2002.

19. Ban K, Peng Z and Kozar RA: Inhibition of ERK1/2 worsens intestinal ischemia/reperfusion injury. PLoS One 8: e76790, 2013.

20. Zhou W, Li S, Wan N, Zhang Z, Guo R and Chen B: Effects of various degrees of oxidative stress induced by intermittent hypoxia in rat myocardial tissues. Respirology 17: 821-829, 2012.

21. Yuan G, Khan SA, Luo W, Nanduri J, Semenza GL and Prabhakar NR: Hypoxia-inducible factor 1 mediates increased expression of NADPH oxidase-2 in response to intermittent hypoxia. J Cell Physiol 226: 2925-2933, 2011.

22. Nanduri J, Vaddi DR, Khan SA, Wang N, Makarenko V, Semenza GL and Prabhakar NR: HIF-1 $\alpha$ activation by intermittent hypoxia requires NADPH oxidase stimulation by xanthine oxidase. PLoS One 10: e0119762, 2015.

23. Yuan G, Nanduri J, Khan S, Semenza GL and Prabhakar NR: Induction of HIF-1alpha expression by intermittent hypoxia: Involvement of NADPH oxidase, $\mathrm{Ca}^{2+}$ signaling, prolyl hydroxylases, and mTOR. J Cell Physiol 217: 674-685, 2008.

24. Nanduri J, Yuan G, Kumar GK, Semenza GL and Prabhakar NR: Transcriptional responses to intermittent hypoxia. Respir Physiol Neurobiol 164: 277-281, 2008.

25. Peng YJ, Yuan G, Ramakrishnan D, Sharma SD, Bosch-Marce M, Kumar GK, Semenza GL and Prabhakar NR: Heterozygous HIF-1alpha deficiency impairs carotid body-mediated systemic responses and reactive oxygen species generation in mice exposed to intermittent hypoxia. J Physiol 577: 705-716, 2006. 
26. Ryan S, Taylor CT and McNicholas WT: Systemic inflammation: A key factor in the pathogenesis of cardiovascular complications in obstructive sleep apnoea syndrome? Thorax 64: 631-636, 2009.

27. Ryan S, McNicholas WT and Taylor CT: A critical role for p38 map kinase in NF-kappaB signaling during intermittent hypoxia/reoxygenation. Biochem Biophys Res Commun 355: 728-733, 2007

28. Greenberg HE, Sica AL, Scharf SM and Ruggiero DA Expression of c-fos in the rat brainstem after chronic intermittent hypoxia. Brain Res 816: 638-645, 1999.

29. Peterson LW and Artis D: Intestinal epithelial cells: Regulators of barrier function and immune homeostasis. Nat Rev Immunol 14: 141-153, 2014.

30. Hering NA, Fromm M and Schulzke JD: Determinants of colonic barrier function in inflammatory bowel disease and potential therapeutics. J Physiol 590: 1035-1044, 2012.

31. Saeedi B, Kendrick A, Schwisow K, Bayless A, Colgan S and Glover L: A role for hypoxia inducible factor in the junctional integrity and barrier function of intestinal epithelial cells (60.1). FASEB J 28: S60.1, 2014.

32. Furuta GT, Turner JR, Taylor CT, Hershberg RM, Comerford K, Narravula S, Podolsky DK and Colgan SP Hypoxia-inducible factor 1-dependent induction of intestinal trefoil factor protects barrier function during hypoxia. J Exp Med 193: 1027-1034, 2001.

33. Guma M, Stepniak D, Shaked H, Spehlmann ME, Shenouda S, Cheroutre H, Vicente-Suarez I, Eckmann L, Kagnoff MF and Karin M: Constitutive intestinal NF- $\kappa \mathrm{B}$ does not trigger destructive inflammation unless accompanied by MAPK activation. J Exp Med 208: 1889-1900, 2011.
34. Fischer A, Gluth M, Pape UF, Wiedenmann B, Theuring F and Baumgart DC: Adalimumab prevents barrier dysfunction and antagonizes distinct effects of TNF- $\alpha$ on tight junction proteins and signaling pathways in intestinal epithelial cells. Am J Physiol Gastrointest Liver Physiol 304: G970-G979, 2013.

35. Ye D, Ma I and Ma TY: Molecular mechanism of tumor necrosis factor-alpha modulation of intestinal epithelial tight junction barrier. Am J Physiol Gastrointest Liver Physiol 290: G496-G504, 2006.

36. Tang Y, Clayburgh DR, Mittal N, Goretsky T, Dirisina R, Zhang Z Kron M, Ivancic D, Katzman RB, Grimm G, et al: Epithelial NF-kappaB enhances transmucosal fluid movement by altering tight junction protein composition after $\mathrm{T}$ cell activation. Am J Pathol 176: 158-167, 2010.

37. Chen ML, Ge Z, Fox JG and Schauer DB: Disruption of tight junctions and induction of proinflammatory cytokine responses in colonic epithelial cells by Campylobacter jejuni. Infect Immun 74: 6581-6589, 2006

38. Al-Sadi R, Ye D, Boivin M, Guo S, Hashimi M, Ereifej L and Ma TY: Interleukin-6 modulation of intestinal epithelial tight junction permeability is mediated by JNK pathway activation of claudin-2 gene. PLoS One 9: e85345, 2014.

39. Taylor CT and Colgan SP: Hypoxia and gastrointestinal disease. J Mol Med Berl 85: 1295-1300, 2007.

40. Colgan SP and Taylor CT: Hypoxia: An alarm signal during intestinal inflammation. Nat Rev Gastroenterol Hepatol 7: 281-287, 2010.

41. Eltzschig HK, Carmeliet P and Carmeliet P: Hypoxia and inflammation. N Engl J Med 364: 656-665, 2011.

42. Sitkovsky M and Lukashev D: Regulation of immune cells by local-tissue oxygen tension: HIF1 $\alpha$ and adenosine receptors. Nat Rev Immunol 5: 712-721, 2005. 